\title{
Supporting a Class' Development in Teamwork
}

\author{
Patricia K. Sheridan ${ }^{1,2}$, Navid Korhani ${ }^{3}$, Doug Reeve ${ }^{1,2}$, and Greg Evans ${ }^{1,2}$ \\ ${ }^{1}$ Institute for Leadership Education in Engineering, ${ }^{2}$ Dept. of Chemical Engineering and Applied Chemistry, \\ ${ }^{3}$ Division of Engineering Science \\ patricia.sheridan@utoronto.ca
}

\begin{abstract}
This paper presents the design and initial adoption of a new class-level interface that has been added to the on-line Team-effectiveness Learning System (TELS) at the University of Toronto. TELS is an on-line self-and peer-assessment system that allows students to provide intra-team feedback within their project teams. This classlevel interface responds to instructor interest in having a better way of assessing and supporting their class' performance in teamwork as a whole. The interface allows an instructor to assess whether the class has demonstrated sufficient proficiency at teamwork, and/or which areas the class needs to improve to demonstrate proficiency.

Launched in September 2015, the class-level interface is currently being used in 5 courses. Preliminary findings indicate the feature to be a useful tool in supporting instructors to tailor their teamwork instruction and activities to the needs of the class.
\end{abstract}

Keywords: team-effectiveness, team-based projects, supporting student teams, online tools, graduate attribute assessment.

\section{INTRODUCTION}

This paper presents the design and use of a new classlevel diagnostic interface that has been added to the on-line Team-effectiveness Learning System (TELS) at the University of Toronto. TELS is a tool that supports teambased project courses by facilitating the development of individual team-effectiveness competencies in students within their teams [1], and by providing instructors with information as to how their teams are performing [2]. Instructors already using the TELS in their courses saw the system as beneficial for student growth, but they also saw opportunities for them to get a better understanding of their class' ability to demonstrate proficiency in teamwork in a way they couldn't through simply observing the teams [3]. As a result, TELS has now developed a class-level diagnostic interface to let instructors see how their students, in aggregate, perform as team members.

Instructors with team-based projects in their courses were interested in the development of this interface for two reasons. Firstly, these instructors wanted to find ways to better support their classes. Instruction of teamwork happens implicitly and explicitly over multiple courses and multiple years, often with different models of effective teamwork being presented based on the course content and instructor preferences. As a result, when faced with a new class at the start of a team-project, instructors were interested in knowing how to best support their students to succeed in the project in their course. We interpreted this interest in two ways: 1) instructors wanted to know in which aspects of teamwork their students needed instruction or reminders of effective strategies, and 2) instructors were interested in knowing which students in their class may be in need of extra support to develop their skills to be an effective team member.

Secondly, these instructors wanted to be able to assess whether their class, in aggregate, demonstrated proficiency in teamwork during their course project. Instructors were interested in using the online system to measure and evaluate whether their class had demonstrated proficiency along the graduate attribute of teamwork. We interpreted this interest in two ways: 1) instructors wanted to be able to determine if their specific class demonstrated proficiency in teamwork, and 2) departments or divisions responsible for accreditation might be interested in determining whether a cohort demonstrated proficiency in teamwork across the team experiences in their undergraduate education.

As a result, we designed the class-level diagnostic interface for TELS to accomplish these instructor interests in three ways: 1) through providing a class performance history that demonstrates previous performance in teamwork in aggregate, 2) through providing instructors a summary of individual student performance in teamwork over their previous team-based projects, and 3) through providing instructors with recommended teamwork topics to cover in their instruction.

\section{TEAM-EFFECTIVENESS LEARNING SYSTEM (TELS)}

Before presenting the class-level diagnostic interface, it is important to describe the team-effectiveness learning system (TELS) in which it is housed, and how this system is used. TELS is an on-line self- and peer-assessment system that allows students to provide intra-team feedback within their project teams. TELS has been presented previously at CEEA outlining its 12-competency inventory of team-member effectiveness [1], and the team-level 
diagnostics available to instructors to better support teams in their growth to become more effective [2].

Twice during a team-based project students provide feedback to themselves and their peers on their teamwork performance in the project. Students provide this feedback on TELS using a 12-competency inventory called the teameffectiveness inventory (TEI) [1]. These 12 competencies are divided equally among three aspects of teamwork determined to be important for a team-member to engage effectively with their team - organizational competencies, relational competencies, and communication competencies. The data from these self- and peerassessments is then compiled and presented to students to guide their development as team members, and to instructors to support teams within their courses.

The TELS is currently used in 7 classes at the University of Toronto. With two assessments per course, and students using TELS in multiple courses, we were accruing a substantial amount of data about individual student's performance in teamwork over time. This, in combination with the instructor interests discussed in the introduction, motivated our use of this longitudinal data to develop a way to better support student learning and report on a class' performance in teamwork.

\section{CLASS-LEVEL INTERFACE}

The class-level interface uses the students' self- and peer-assessments provided within TELS to create a series of diagnostic visuals that support an instructor in understanding how their class, and the individual students within it, perform in teamwork. These visuals allow an instructor to assess whether the class has demonstrated sufficient proficiency at teamwork, and/or which areas the class needs to improve on to demonstrate proficiency.

Diagnostic information is provided to an instructor on three levels:

1) the class' aggregate longitudinal performance in teamwork,

2) an individual student's longitudinal performance in teamwork, and

3) a class' areas of strength and weakness based on previous and current performance.

Please note that all visuals and examples contained within this paper are of hypothetical students and classes.

\subsection{Class Performance History}

The class performance history aggregates student assessment from past teamwork experiences to create a representation of how the class as a whole performs in teamwork. It identifies for an instructor the distribution of student performance in teamwork and differentiates for them the students who are and are not struggling with teamwork. Figure 1 provides instructors with a summary of the class' aspect-level strength and weakness as well as a listing of each student's average performance across the 12 competencies. Each of the table headings in Figure 1 are sortable, allowing an instructor to easily identify those students who have previously performed well or poorly at teamwork, or those students who have had alerts raised from the discrepancies between their self- and peerassessments [2]. This information affords an instructor an understanding of their class' makeup in terms of teamwork performance, and may inform how they choose to design the teamwork experience.

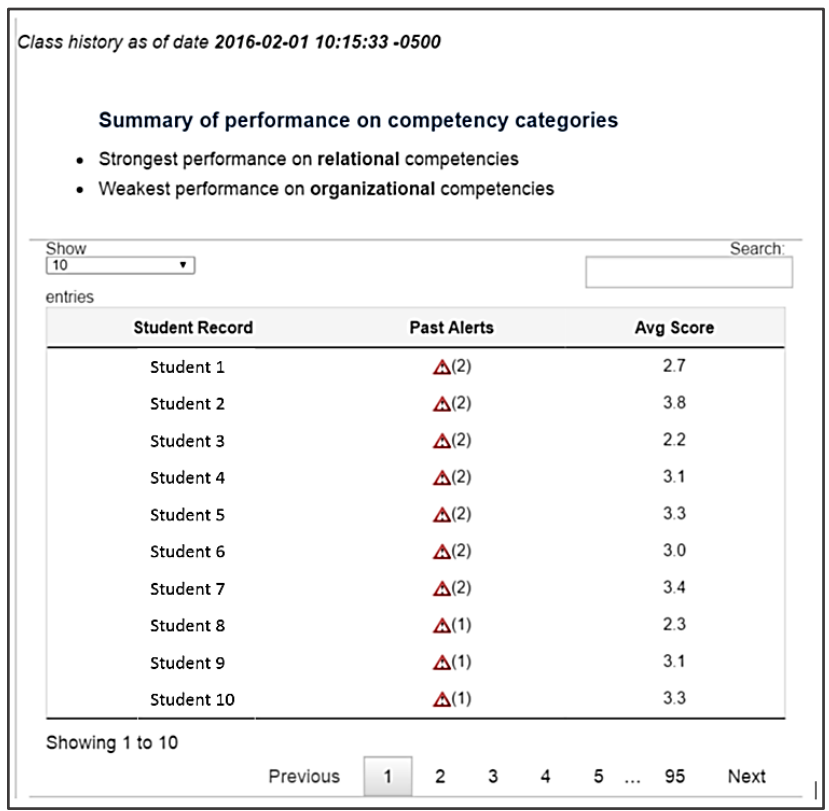

Fig.1. Class history summary that provides an instructor with the strength and weakness of the class as well as a sortable table of individual student performance.

The class performance history also provides the instructor with a sense of the spread of performance-levels of the students in their class, as shown in Fig. 2. This will inform an instructor as to whether the class as whole has a similar performance level (top and bottom 20 percentiles are similar to the average), or whether they are dealing with a class of substantially varied performance as shown in Fig. 2. If the class has a substantially varied performance this may influence the way in which instructors choose to form the teams in their current course, and whether they use this information to support student teams differently based on need.

Figure 2 also shows the average performance of the class along each of the three aspects of teamwork. This will inform an instructor as to whether there is one aspect of teamwork that is dragging down the class' performance, or whether they are performing equally across all aspects. This can inform the type of teamwork instruction an instructor might choose to give at the outset of a new project. As a result, this information is used to recommend 
teamwork topics for instructors to consider discussing, as described in Section 3.3.

Class Average Scores(range from 1 to 4 )

\begin{tabular}{|l|l|}
\hline Top 20 Percentile & 3.7 \\
\hline Mean & 3.0 \\
\hline Bottom 20 Percentile & 2.1 \\
\hline Organizational Competencies & 2.8 \\
\hline Communication Competencies & 3.0 \\
\hline Relational Competencies & 3.2 \\
\hline $\begin{array}{l}\text { Click on each competency category to see the list of the } \\
\text { competencies and their scores }\end{array}$
\end{tabular}

Fig. 2. Class average scores table that provides an instructor with a sense of the spread of teamwork performance across the class, and across the aspects of the TEI.

\subsection{Student Record of Teamwork}

The student record of teamwork provides a visual representation to the instructor as to how an individual student in their course has performed in teamwork over their past team-based projects. Two visuals, Figs. 3 and 4, along with a quick-glance summary, Fig. 5, are provided to the instructor that demonstrate how the student has performed relative to their class over time.

The first visual, the summary of teamwork performance in Fig. 3, provides an instructor with a representation of the student's aggregate performance along all 12 competencies of the TEI from their previous assessments. As can be seen in Fig. 3, the student's performance over the four courses is graphed relative to the class average of teamwork performance. We chose to represent student performance relative to the class to account for class-level improvements in proficiency. In Fig. 3, we can see that the student has improved their performance in teamwork relative to their class over the four courses. For an instructor this would identify a student who is improving, and would not intuitively need to be of concern. If however, we saw the slope of the student's performance decreasing over time, an instructor would be able to identify this as a student struggling with teamwork and in need of extra support.

To determine how to best support that student, the instructor can find more information in the average competency scores graph, Fig. 4, which outlines the student's performance relative to the class average across the three aspects measured in the TEI. In the example in Fig. 4, an instructor can see that the student continues to struggle with relational competency in teamwork, and in the last course has had an issue with organizational competency. With this information, an instructor could recommend to the student that they try out some new techniques to enhance their competency along these aspects, or they could refer them to the online lessons on TELS for those two aspects [1].

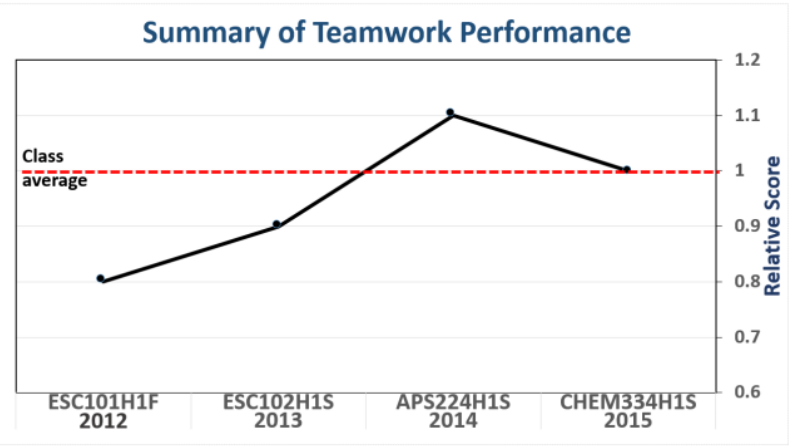

Fig. 3. Aggregate summary of student performance in teamwork demonstrated relative to class average over multiple courses.

\section{Average Competency Scores}

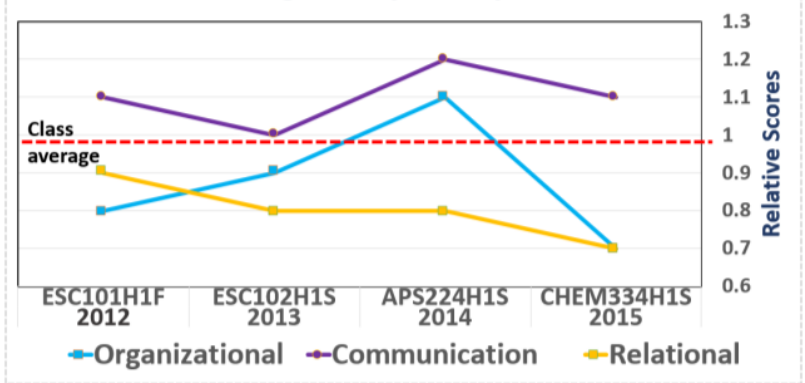

Fig. 4. Aspect-level summary of student performance in teamwork demonstrated relative to class average over multiple courses.

If an instructor, however, is looking for an abbreviated evaluation of a student's performance, they can look at the quick-summary available for each student, as shown in Fig. 5. From this summary, an instructor can get an at-a-glance listing of the student's strengths and weaknesses, as well as whether the student has had any team-level alerts flagged with their self- and peer-assessments in previous courses. Team-level alerts identify to an instructor the inconsistencies between self- and peer-assessments for that student in specific past courses [2]. This information might alert an instructor to a student who has continually struggled with teamwork, or to a student who perceives the value of teamwork very differently than their team members.

\subsection{Recommended Teamwork Topics}

The recommended teamwork topics lists for an instructor the competencies of the inventory along which the class as a whole were assessed as performing the best 
and the worst. These are identified as recommended teamwork topics as they are the competencies along which to commend the class for their demonstrated proficiency (strengths) and the competencies along which the instructor needs to provide more instruction (weaknesses). The two competencies along which the class had the highest ratings are listed as the class' strengths, and the two competencies along which the class had the lowest ratings are listed as the class' weaknesses, Fig. 6. This can alert an instructor to the aspects of teamwork which the students understand and are readily applying, as well as to the aspects which the students are struggling to succeed in.

\section{Team-level Alerts:}

\begin{tabular}{|l|l|}
\hline Course 1 & rated themselves higher than their peers \\
\hline Course 2 & rated themselves higher than their peers \\
\hline
\end{tabular}

\section{Summary of Performance}

- Strongest performance on relational competencies

- Weakest performance on organizational competencies

\section{Strengths}

1. Show respect for team members

2. Raise contentious issues in a constructive way

\section{Weaknesses}

1. Attend team meetings prepared

2. Promote productive discussion

Fig. 5. Quick-summary listing of a student's performance trends in teamwork over multiple courses.

Strengths
- Show respect for team members
Wisten and pay attention to team members
- Do their fair share of the work (\%46)
- Deliver their work on time (\%43)
portion of the students who have this competency in the bottom three of their list

Fig.6. Listing of a class' top two strengths and bottom two weaknesses.

In addition to information on the highest and lowest rated competencies, an instructor can also find out how frequently a competency is listed as an individual student's weakness (lowest three rated competencies by peers [1]).
This information is shown for each aspect, as shown for the communication competency aspect in Fig. 7. Here, an instructor can see the competencies associated with the aspect, the class' average score along each competency, and what percent of the class had that competency rated in their bottom three competencies (listed as a weakness).

We chose to provide instructors with both pieces of information as the absolute average assessment may not be representative of whether a particular competency is a shared weakness among the class.

\begin{tabular}{|lcc|}
\hline \multicolumn{2}{|c|}{ Communication Competencies } \\
\hline \multicolumn{1}{|c|}{ Competency } & $\begin{array}{c}\text { Avg } \\
\text { Score }\end{array}$ & $\begin{array}{c}\text { Weakness } \\
\text { Prevalence }\end{array}$ \\
$\begin{array}{l}\text { Exchange information in a timely } \\
\text { manner }\end{array}$ & 2.8 & $\% 18$ \\
$\begin{array}{l}\text { Raise contentious issues in a } \\
\text { constructive way }\end{array}$ & 2.9 & $\% 36$ \\
$\begin{array}{l}\text { Promote productive discussion } \\
\text { Openly express ideas and opinions }\end{array}$ & 3.0 & $\% 32$ \\
\hline
\end{tabular}

Fig.7. Aspect-level competency information as to the average rating of the competency for the class in aggregate, as well as the prevalence of the competency in a student's list of weaknesses.

\section{ADOPTION AND USAGE}

Launched in September 2015, the class-level interface is currently being used in 5 courses, with approximately 50 course instructors and teaching assistants.

Some instructors anecdotally provided feedback on the interface to the authors. These instructors found the student record of teamwork and the recommended teamwork topics to be the most useful components of the class-level interface. They expressed concerns over how the classperformance history might influence an instructor to have a pre-conceived notion about the performance of their class that may not represent student growth over time. As a result, this aspect was seen of as the least useful.

In courses, the interface has been primarily used to tailor class-level instruction to the needs of a class depending on trends from previous teamwork assessments.

For example, one lecturer used the class-level interface to tailor a class' lecture on teamwork to the needs of the class. The class had previously used the TELS system to provide self- and peer-assessments, and class-level data from those assessments was available to the lecturer. The lecturer used this information to tailor the lecture on teamwork strategies to the needs of the class. They opened by commending the class on what they had done well (highlighting top two strengths of the class as a whole). They then dedicated the remainder of the lecture to providing teaming strategies to the class related to the two competencies identified as the class' weaknesses. As a result of this, students were able to receive a teamwork 
lecture that addressed the aspects of teamwork with which the class as a whole was struggling the most.

\section{DISCUSSION}

Through our development and testing of the interface, one concern that has been raised is around the privacy implications of passing student performance information between courses. While this information is intended as a way of providing an instructor with the ability to tailor their instruction to the needs of the students, whether instructors should know this information from past courses has been a question. We are currently exploring ways to ensure that student privacy is not compromised while still providing instructors the ability to best meet the needs of their class.

\section{CONCLUSION}

A class-level interface has been designed and incorporated into the Team-effectiveness Learning System to provide instructors with information to guide the way in which they design their class' teamwork instruction. The interface provides information on the distribution of student-performance across the aspects of teamwork and across the students in the class, as well as recommended teamwork topics for instruction. The recommended teamwork topics approach to tailoring instruction has proved the most useful component to instructors thus far.

While the interface is solely used for instructor information at this point, there are indications that the interface could be used to provide information for graduate attribute reporting. For cohorts graduating, this interface could provide information as to how the cohort performs at teamwork and whether the cohort has demonstrated sufficient proficiency.

\section{Acknowledgements}

This research supported in part by the Ontario Graduate Scholarship, and the University of Toronto Instructional Technology Innovation Fund. The authors would also like to thank all the instructors who provided us feedback on the new interface.

\section{References}

[1] P. K. Sheridan, P. M. To, G. J. Evans and D. W. Reeve, "An On-line Team-effectiveness Learning System," in Proceedings of the Canadian Engineering Education Annual Conference, Canmore, AB, 2014.

[2] P. K. Sheridan, A. Malone, D. Reeve and G. Evans, "Seeing into your Teams: An Instructor Interface to Support Team Learning," in Canadian Engineering Education Association Annual Conference, Hamilton, ON, 2015.

[3] P. K. Sheridan, D. Reeve and G. Evans, "Understanding Teaching Assistants' Assessment of Individual Teamwork Performance," in American Society for Engineering Education Annual Conference and Exhibition, Indianapolis, IN, 2014. 